\title{
ARRAYS AND BROOKS
}

B. T. BENNETT and R. B. POTTS

(Received 21 March 1966)

\section{Constant-sum arrays}

Consider an $m \times n$ rectangular array whose $m$ rows are permutations of $1,2, \cdots, n$. Such an array will be called a constant-sum array if the sum of the elements in each column is the same (and equal to $\frac{1}{2} m(n+1)$ ). An example of a $3 \times 9$ constant-sum array is

$$
\left[\begin{array}{lllllllll}
1 & 2 & 3 & 4 & 5 & 6 & 7 & 8 & 9 \\
8 & 6 & 9 & 3 & 1 & 7 & 4 & 2 & 5 \\
6 & 7 & 3 & 8 & 9 & 2 & 4 & 5 & 1
\end{array}\right] .
$$

In contrast to a Latin rectangle, elements in the same column of a constantsum array may be equal. It will be convenient to assume arrays normalised in the sense that the columns are arranged so that, as in (1), the first row is in the standard order $1,2, \cdots, n$.

The combinatorial problem of counting and classifying constant-sum arrays can be described in a number of ways. One may appeal to the theory of partitions and regard each column as a partition of $\frac{1}{2} m(n+1)$ into $m$ parts with no part greater than $n$. A constant-sum array is obtained from the partitions by choosing and ordering them so that the rows are permutations of $1,2, \cdots, n$. The number of partitions when $m=3$ and $n$ is odd, for example, is equal to the greatest integer not greater than $\frac{1}{8}(n+1)^{2}+\frac{1}{2}$.

Alternatively the problem can be described as the following "log problem". First consider $m$ differently coloured logs each of length $\frac{1}{2} n(n+1)$ units, and then suppose that each $\log$ is cut into $n$ pieces of lengths $1,2, \cdots, n$ units. The problem is then to form from the $m n$ pieces $n$ logs of equal length, each consisting of $m$ differently coloured pieces.

There are no constant-sum arrays with an odd number of rows and an even number of columns. Since most of this paper will be confined to three-rowed arrays, it is convenient to restrict the number of columns to be odd and introduce the notion of zero-sum arrays. 


\section{Zero-sum arrays}

Consider an $m \times(2 n+1)$ rectangular array whose $m$ rows are permutations of the $2 n+1$ integers $-n,-n+1, \cdots, n-1, n$. Such an array will be called a zero-sum array if the sum of the elements in each column is zero. An example of a $\mathbf{3} \times \mathbf{9}$ zero-sum array is

$$
\left[\begin{array}{rrrrrrrrr}
-4 & -3 & -2 & -1 & 0 & 1 & 2 & 3 & 4 \\
3 & 1 & 4 & -2 & -4 & 2 & -1 & -3 & 0 \\
1 & 2 & -2 & 3 & 4 & -3 & -1 & 0 & -4
\end{array}\right]
$$

A zero-sum array will always be assumed to be normalised in the sense that the first row is in the standard order $-n,-n+1, \cdots, n-1, n$. From a zero-sum array a corresponding constant-sum array is obtained by adding $n+1$ to each element.

It is easy to prove the existence of a zero-sum array for any $m, n$. For $m=2$, a unique $2 \times(2 n+1)$ zero-sum array is obtained:

$$
\left[\begin{array}{rrlrr}
-n & -n+1 & \cdots & n-1 & n \\
n & n-1 & \cdots & -n+1 & -n
\end{array}\right]
$$

For $m=3$, an example of a $3 \times(2 n+1)$ zero-sum array is

(4) $\left[\begin{array}{rrrlrrrcc}-n & -n+1 & -n+2 & \cdots & 0 & 1 & 2 & \cdots & n \\ 0 & 1 & 2 & \cdots & n & -n & -n+1 & \cdots & -1 \\ n & n-2 & n-4 & \cdots & -n & n-1 & n-3 & \cdots & -n+1\end{array}\right]$.

By combining these 2 and 3 rowed arrays, an $m \times(2 n+1)$ zero-sum array can be obtained for any $m, n$.

\section{Three-rowed zero-sum arrays}

The enumeration and classification of the zero-sum arrays for any $m, n$ is exceedingly difficult and it is the purpose of this paper to report some results for arrays with only three rows. The values of

$$
N(n)=\text { number of } 3 \times(2 n+1) \text { zero-sum arrays }
$$

have been calculated for small values of $n$ using a CDC3200 computer and the results are given in the second row of Table 1. Some of the zero-sum arrays for small $n$ are exhibited in Table 2 . As is often the case with combinatorial problems, the value of $N(n)$ increases rapidly with $n$ and even computer evaluation soon becomes prohibitive.

Permuting the rows of an array or changing the sign of each element 
TABLE 1. The number $N(n)$ of different $3 \times(2 n+1)$ zero-sum arrays, and the breakdown of $N(n)$ into numbers of arrays in classes $C_{1}$ to $C_{6}$.

\begin{tabular}{|c|c|c|c|c|c|c|c|c|c|}
\hline & $n$ & 0 & 1 & 2 & 3 & 4 & 5 & 6 & 7 \\
\hline & $N(n)$ & 1 & 2 & 6 & 28 & 244 & 2,544 & 35,600 & 659,632 \\
\hline$C_{1}$ & $(\times 12)$ & & 0 & 0 & I & 15 & 200 & 2,924 & 54,774 \\
\hline$C_{2}$ & $(\times 6)$ & & 0 & 0 & 0 & 1 & 0 & 0 & 26 \\
\hline$C_{\mathrm{s}}$ & $(\times 6)$ & & 0 & 1 & 2 & 7 & 24 & 80 & 306 \\
\hline$C_{4}$ & $\left(\begin{array}{ll}\times 4\end{array}\right)$ & & 0 & 0 & 0 & 2 & 0 & 8 & 82 \\
\hline$C_{8}$ & $(\times 2)$ & & 0 & 0 & 1 & 0 & 0 & 0 & 0 \\
\hline$c_{\bullet}$ & $\left(\begin{array}{ll}\times 2\end{array}\right)$ & & 1 & 0 & 1 & 4 & 0 & 0 & 12 \\
\hline
\end{tabular}

TABLE 2. Examples of $3 \times(2 n+1)$ zero-sum arrays.

(a) $n=0, N(0)=1$.

$$
\left[\begin{array}{l}
0 \\
0 \\
0
\end{array}\right]
$$

(i)

(b) $n=1, N(1)=2$.

$$
\left[\begin{array}{rrr}
-1 & 0 & 1 \\
0 & 1 & -1 \\
1 & -1 & 0
\end{array}\right]
$$

(i) $\left[\begin{array}{rrr}-1 & 0 & 1 \\ 1 & -1 & 0 \\ 0 & 1 & -1\end{array}\right]$

(ii)

(c) $n=2, N(2)=6$.

$\left[\begin{array}{rrrrr}-2 & -1 & 0 & 1 & 2 \\ 0 & 1 & 2 & -2 & -1 \\ 2 & 0 & -2 & 1 & -1\end{array}\right] \quad\left[\begin{array}{rrrrr}-2 & -1 & 0 & 1 & 2 \\ 1 & 2 & -2 & -1 & 0 \\ 1 & -1 & 2 & 0 & -2\end{array}\right]$

(i)

$\left[\begin{array}{rrrrr}-2 & -1 & 0 & 1 & 2 \\ 2 & 0 & -2 & 1 & -1 \\ 0 & 1 & 2 & -2 & -1\end{array}\right]$

(iii)

$\left[\begin{array}{rrrrr}-2 & -1 & 0 & 1 & 2 \\ 2 & -1 & 1 & -2 & 0 \\ 0 & 2 & -1 & 1 & -2\end{array}\right]$

(v)

$\left[\begin{array}{rrrrr}-2 & -1 & 0 & 1 & 2 \\ 1 & -1 & 2 & 0 & -2 \\ 1 & 2 & -2 & -1 & 0\end{array}\right]$

(iv)

$\left[\begin{array}{rrrrr}-2 & -1 & 0 & 1 & 2 \\ 0 & 2 & -1 & 1 & -2 \\ 2 & -1 & 1 & -2 & 0\end{array}\right]$

(vi)

does not alter the zero-sum property. From the array (2), for example, the zero-sum array

(6) $\quad\left[\begin{array}{rrrrrrrrr}-4 & -3 & -2 & -1 & 0 & 1 & 2 & 3 & 4 \\ 0 & 2 & 4 & -1 & -3 & 3 & 1 & -2 & -4 \\ 4 & 1 & -2 & 2 & 3 & -4 & -3 & -1 & 0\end{array}\right]$ 
is obtained by interchanging rows I and III (and then normalising), and the zero-sum array

$$
\left[\begin{array}{rrrrrrrrr}
-4 & -3 & -2 & -1 & 0 & 1 & 2 & 3 & 4 \\
0 & 3 & 1 & -2 & 4 & 2 & -4 & -1 & -3 \\
4 & 0 & 1 & 3 & -4 & -3 & 2 & -2 & -1
\end{array}\right]
$$

is obtained by changing signs throughout (and then nornalising). The six permutations of the three rows I, II, III, together with the sign changes, allow the possibility of a class of twelve related $3 \times(2 n+1)$ zero-sum arrays. The possible operations are listed in the second column of Table 3 , but not

TABLE 3. Symmetries of a regular hexagon and related operations on a zero-sum array.

\begin{tabular}{|c|c|}
\hline symmetry & operation \\
\hline$I$ & (I) (II) (III) \\
\hline $\boldsymbol{R}$ & $-($ I III II $)$ \\
\hline$R^{2}$ & (I II III) \\
\hline$R^{s}$ & $-(I)(I I)(I I I)$ \\
\hline$R^{4}$ & (I III II) \\
\hline$R^{\mathbf{b}}$ & $-($ I II III $)$ \\
\hline$H$ & $-(\mathrm{I}$ II $)(\mathrm{III})$ \\
\hline$H R$ & (I) (II III) \\
\hline$H R^{2}$ & $-(\mathrm{I}$ III $)(\mathrm{II})$ \\
\hline$H R^{3}$ & $(\mathrm{I}$ II $)(\mathrm{III})$ \\
\hline$H R^{4}$ & $-(\mathrm{I})$ (II III) \\
\hline$H R^{6}$ & $(\mathrm{I} I I I)(I I)$ \\
\hline
\end{tabular}

all the related arrays need be different. A classification of the $3 \times(2 n+1)$ zero-sum arrays is facilitated by interpreting them as solutions to a problem similar to two classical chess-board problems.

\section{Rook, queen, and brook problem}

The solution of the general rook (or queen) problem requires the determination of the number of ways $n$ rooks (or queens) can be placed on an $n \times n$ chess-board so that no rook (or queen) can take any other [1]. Alternatively the rook problem can be described as the enumeration of the number of ways $n$ objects can be placed on cells of an $n \times n$ square lattice such that no two are in the same row or column. For the queen froblem, no two of the objects can lie in the same row, column or either diagonal. The rook problem is trivial, as there are simply $n$ ! ways of placing the rooks on the board. The solutions to the problem can be classified using properties of the dihedral group of order $8-$ the group of symmetries of a square [1]. 
The rook problem is not so trivial when the board is restricted by elimination of some of the squares [2]. The queen problem is as yet unsolved although the solutions for small $n$ have been enumerated and classified [1].

For present purposes it is convenient to invent a new chess piece the brook - which is able to move on any row or column and also in one diagonal direction. The brook might be described as a rook plus a halfbishop and is intermediate between a rook and a queen. The chess-board is assumed to be a $(2 n+1) \times(2 n+1)$ board which is restricted by excluding $n(n+1)$ squares from the NE and SW corners (for $n=4$, Fig. 1 illustrates the $9 \times 9$ board restricted by exclusion of the 20 shaded squares). The brook is free to move N, S, E, W, NW or SE as indicated in Fig. 1 by the dotted lines. These lines form the dual lattice which when distorted

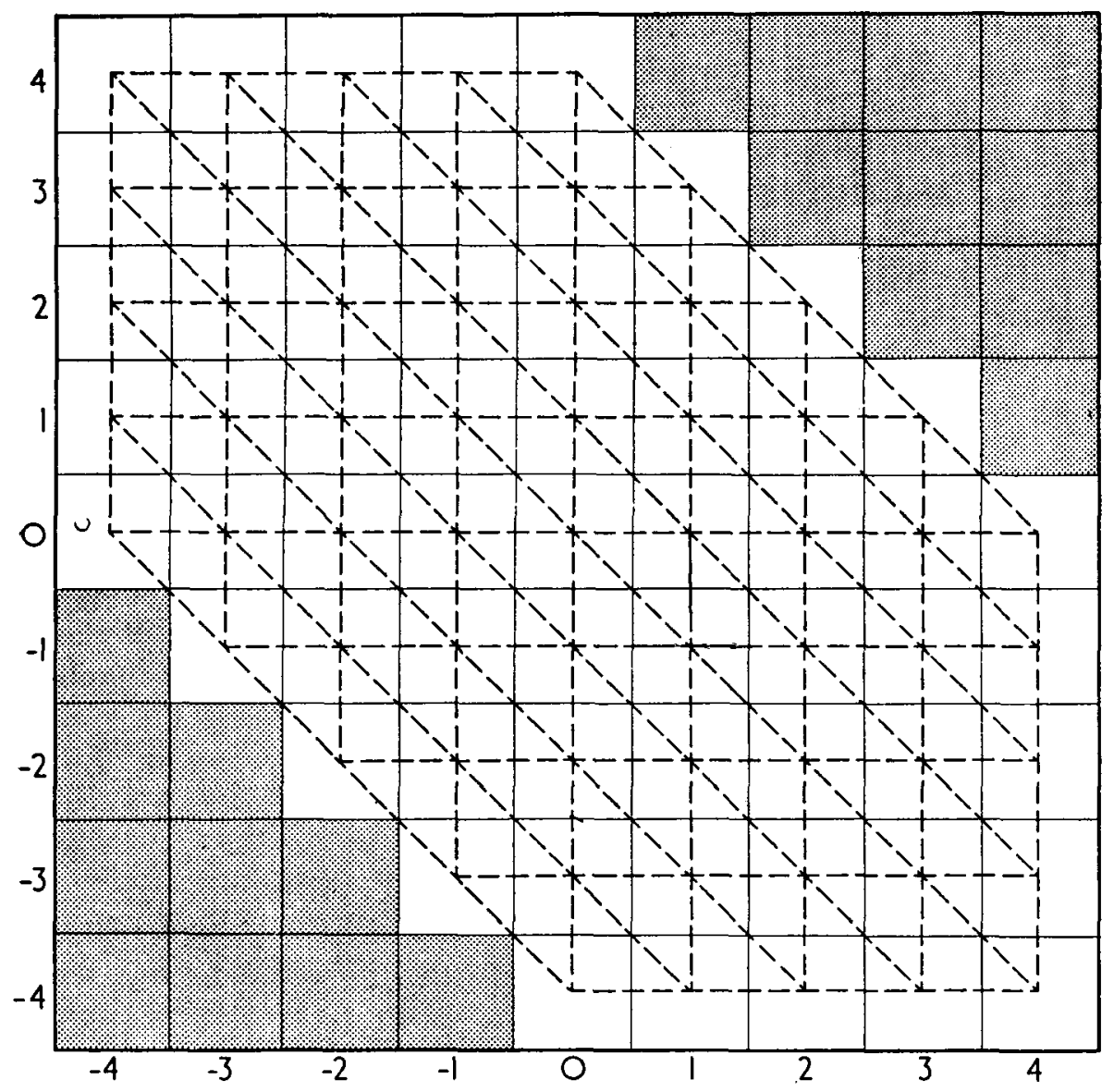

Fig. 1. A $9 \times 9$ restricted chess board. The 'brook' is able to move vertically, horizontally or in one diagonal direction as indicated by the dotted lines. 


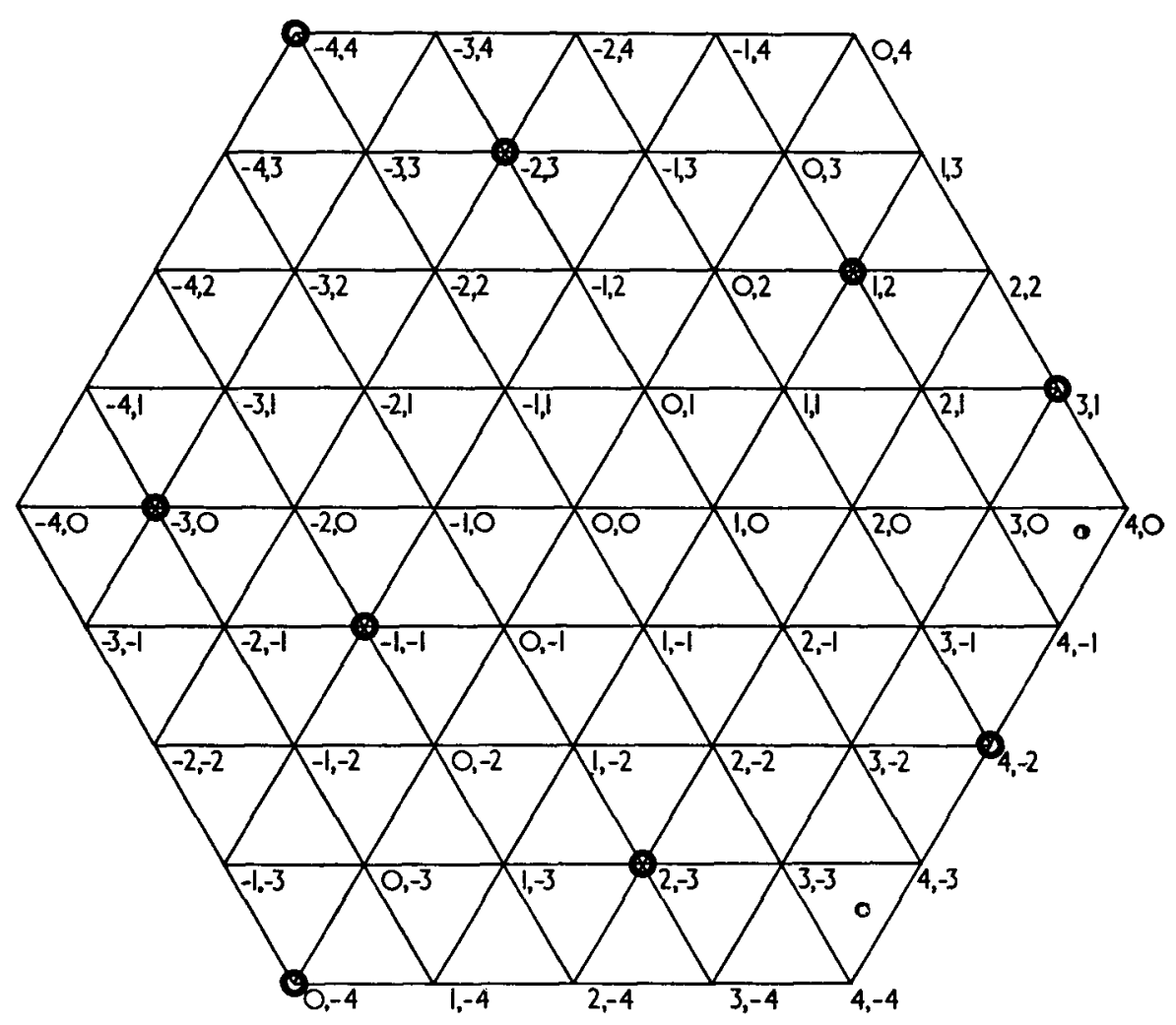

Fig. 2. A 4 triboard with a solution to the brook problem.

is seen to be a portion of a triangular lattice bounded by a regular hexagon with edge of length $n$ units. Such a figure will, for brevity, be called an $n$ triboard and it is the dual of the so-called $n$ tricolour board used in the game of tricolour [1]. Fig. 2 illustrates a 4 triboard. The brook problem of placing brooks on squares of a restricted chess-board is equivalent to the problem of placing brooks on grid points of a triboard. More precisely, the solution of the brook problem requires the determination of the number of ways $2 n+1$ brooks can be placed on the grid points of an $n$ triboard such that no two brooks lie on the same straight line. Just as the solutions to the rook problem can be classified according to the symmetries of a square, so can the solutions to the brook problem be classified according to the symmetries of a regular hexagon.

\section{Brook problem and zero-sum arrays}

There is a 1-1 correspondence between solutions to the brook problem and zero-sum arrays. Each grid puint of the triboard can be made to 
represent a column of a zero-sum array by using coordinates as indicated in Fig. 2. For example, the first coordinate may be taken as the second element in a column, the second coordinate as the third element in the column, and minus the sum of the coordinates as the first element in the column. The circled grid points in Fig. 2 then represent a solution to the brook problem which corresponds to the zero-sum array (2). Note that the correct normalisation of the array is obtained by starting with the edge joining 0,4 and 4,0 and proceeding to parallel lines across the diagram, downwards and to the left.

\section{Classification of solutions}

The possibility of obtaining a class of twelve related zero-sum arrays by row permutations and change of sign can be interpreted in terms of the symmetries of a regular hexagon. The twelve symmetries (elements of the dihedral group of order 12) are listed in the first column of Table $3, R$ denoting a rotation anticlockwise through $60^{\circ}$ and $H$ a reflection in the horizontal axis [3]. The corresponding operations on the zero-sum arrrays are listed in the second column of Table 3. A solution can be classified according to the largest subgroup of the dihedral group under which the solution is invariant. This classification is given in Table 4, in which solutions invariant under $\{I, H\}$ or $\left\{I, H R^{2}\right\}$, or $\left\{I, H R^{4}\right\}$ have been classed together.

TABLE 4. Classification of solutions.

\begin{tabular}{lll}
\hline class & $\begin{array}{l}\text { number of } \\
\text { solutions }\end{array}$ & subgroup \\
\hline$C_{1}$ & 12 & $\{I\}$ \\
$C_{2}$ & 6 & $\left\{I, R^{3}\right\}$ \\
$C_{3}$ & 6 & $\{I, H\},\left\{I, H R^{2}\right\},\left\{I, H R^{4}\right\}$ \\
$C_{4}$ & 4 & $\left\{I, R^{2}, R^{4}\right\}$ \\
$C_{5}$ & 2 & $\left\{I, R, R^{2}, R^{8}, R^{4}, R^{6}\right\}$ \\
$C_{6}$ & 2 & $\left\{I, R^{2}, R^{4}, H, H R^{2}, H R^{4}\right\}$ \\
\hline
\end{tabular}

Because no two brooks can lie on the same straight line, it is clear that a solution cannot be invariant under $H R, H R^{3}$, or $H R^{5}$, which are reflections in lines perpendicular to opposite edges of the hexagon. The number of solutions in each class, giving a breakdown of the total number $N(n)$, have been calculated using a computer and the results are given in Table 1. The zero-sum array (2) is in class $C_{1}$ as can be verified from the corresponding brook solution which has no symmetry (see Fig. 2). 


\section{Brook polynomials}

Computer calculations have enabled the evaluation, for small $n$, of the generating functions defined by

$$
B_{2 n+1}(x)=\sum_{k=0}^{2 n+1} b_{n k} x^{k}
$$

where $b_{n k}=$ the number of ways $k$ brooks can be placed on the grid points of an $n$ triboard such that no two brooks lie in the same straight line.

The generating functions may be called brook polynomials by analogy with the rook polynomials which play a central role in the analysis of the rook problem [2]. The first five brook polynomials are

$$
\begin{aligned}
& B_{1}(x)=1+x \\
& B_{3}(x)=1+7 x+6 x^{2}+2 x^{3} \\
& B_{5}(x)=1+19 x+87 x^{2}+115 x^{3}+30 x^{4}+6 x^{5} \\
& B_{7}(x)=1+37 x+417 x^{2}+1,783 x^{3}+2,902 x^{4}+1,629 x^{5}+196 x^{6}+28 x^{7} \\
& B_{9}(x)=1+61 x+1,278 x^{2}+11,758 x^{3}+50,465 x^{4}+99,717 x^{5}+84,366 x^{6} \\
& \quad+26,836 x^{7}+2,196 x^{8}+244 x^{9} .
\end{aligned}
$$

From the definition of $b_{n k}$ it is clear that $b_{n 2 n+1}=N(n)$ and $b_{n k}=0$ for $k>2 n+1$. For small $k$ the following results have been obtained, the last two expressions being conjectures based on limited numerical values.

$$
\begin{aligned}
& b_{n 0}=1 \\
& b_{n 1}=3 n^{2}+3 n+1 \\
& b_{n 2}= \frac{1}{2}\left(9 n^{4}+4 n^{3}-n\right) \\
& b_{n 3}=\frac{1}{8}\left(36 n^{6}-60 n^{5}+30 n^{4}+4 n^{3}+8 n^{2}-4 n+1\right)-\frac{1}{8}(-1)^{n} \\
& b_{n 4}=\frac{1}{8}\left(27 n^{8}-144 n^{7}+322 n^{6}-364 n^{5}+198 n^{4}-60 n^{3}+40 n^{2}-27 n+7\right) \\
& \quad \quad-(-1)^{n} \frac{1}{8}\left(3 n^{2}-9 n+7\right) .
\end{aligned}
$$

It has not been found possible to find general formulae for $b_{n k}$, $B_{2 n+1}(x)$, or $N(n)$. The problem of determining these seems to be somewhere intermediate between the trivial rook problem and the unsolved queen problem! 


\section{References}

[1] M. Kraitchik, Mathematical Recreations (G. Allen and Unwin, 1943), Ch. 10.

[2] J. Riordan, An Introduction to Combinatorial Analysis (Wiley, 1958), Ch. 7, 8.

[3] W. Ledermann, Introduction to the Theory of Finite Groups (Oliver and Boyd, 1953), p. 89.

\section{Mathematics Department}

University of Adelaide 